\title{
Revisiting an old issue: Retroactive interference as a function of the degree of original and interpolated learning
}

\author{
KARL-HEINZ BÄUML \\ Universität Regensburg, Regensburg, Germany
}

\begin{abstract}
Prior research has generally shown that the greater the degree of original learning of a list, the greater the amount of retroactive interference that list suffers. In addition, greater learning of interpolated lists produces more retroactive interference. However, in prior research, the degree of learning has typically been confounded with the amount of retrieval practice on the list. Two free-recall experiments are reported in which subjects studied one original list and then $0,1,2,3$, or 4 interpolated lists. The degree of original and of interpolated learning was manipulated by varying exposure time. In Experiment 1, where the typical confounding of retrieval practice and degree of interpolated learning was present, greater interpolated learning induced greater retroactive interference, which is consistent with prior research. However, in Experiment 2, where the degree of interpolated learning was manipulated without concomitant variation in retrieval practice, retroactive interference was the same, whether the interpolated lists had been learned well or poorly. Therefore, greater interpolated learning does not increase the amount of retroactive interference. The results also show that the amount of retroactive interference does not depend on the degree of original learning, in agreement with other work on normal forgetting.
\end{abstract}

Does the forgetting of verbal material depend on the degree to which it is learned? Specifically, does a higher degree of learning induce more forgetting or less forgetting than does a lower degree? It is important to discover the relationship between forgetting and the degree of learning not only for practical reasons but also for theoretical ones. Indeed, the empirical relationship between the two factors will establish a restriction for models of forgetting and thus may help to distinguish between different memory models.

The relationship between the degree of learning and the amount of forgetting has been studied using different experimental designs. Forgetting was examined as a function of the time that passes between learning and test (normal forgetting), as a function of some prior learning (proactive interference), and as a function of some subsequent learning (retroactive interference). Despite the great number of experiments that has been conducted, solid information about the interplay between the degree of learning and forgetting exists only for normal forgetting, based mainly on the work of Slamecka and McElree (1983). Noting a number of problems in earlier studies, these researchers conducted a series of new experiments in which they demonstrated that the degree to which material is learned does not influence the amount of normal forgetting. ${ }^{1}$

The author thanks M. Anderson, D. Macaulay, H. L. Roediger III, and one anonymous referee for their very helpful comments on earlier drafts of the manuscript. Correspondence should be addressed to K.-H. Bäuml, Institut für Psychologie, Universität Regensburg, 93040 Regensburg, Germany (e-mail: heinz@rpss3.psychologie.uni-regensburg.de)
With respect to retroactive interference, the situation is less clear. While some researchers found evidence that a higher degree of original learning is subject to more retroactive interference than is a lower degree (Briggs, 1957; Postman \& Riley, 1959), other researchers found that, in this case, forgetting occurs independently from the degree of learning (Slamecka, 1960). It has even been postulated that a higher degree of learning is associated with less retroactive interference than is a lower degree (Wickelgren, 1977).

Several aspects of the prior experiments might be responsible for the inconsistency in results (see Slamecka $\&$ McElree, 1983). Above all, the inspection of data from many studies showing that retroactive interference varies with the degree of learning raises suspicion about floor effects for the lower degrees of learning. In fact, these floor effects seem to carry the brunt of the dependency, indicating that retroactive interference may not depend on the degree of learning. However, to answer the question in a less tentative way, fresh data, which are reasonably free of the floor effect problem, are necessary.

A related question arises with respect to retroactive interference: Does the amount of forgetting depend on the degree of interpolated learning? Earlier studies found that a higher degree of interpolated learning induced greater interference than did a lower degree (Barnes \& Underwood, 1959; Briggs, 1957; Postman \& Riley, 1959). Recently, however, Anderson, Bjork, and Bjork (1994) have pointed out that this position might be wrong.

All of the studies showing an effect of the degree of interpolated learning used the anticipation procedure during acquisition of the interpolated task. In this procedure, 
subjects must learn to give the response member of a paired associate when presented with the stimulus member of the pair. On each trial, the stimulus member is presented as a cue to which subjects attempt to recall or "anticipate" the associated response. Following the response, feedback is given as to the correct pairing. By cuing recall in this manner, subjects are engaged in retrieval practice with every trial of the learning task.

Using this procedure, the degree of interpolated learning is manipulated by varying the number of study trials for the interpolated task. However, as more study opportunities are given, more prescribed retrieval efforts are also being made, thus increasing the retrieval practice on the interpolated task. On the basis of their recent finding that the retrieval of material can cause long-lasting forgetting of related material (retrieval-induced forgetting), Anderson et al. (1994) have noticed that retrieval practice on the interpolated task may suppress the retrieval of the original task, with a higher amount of retrieval practice leading to a stronger suppression. Thus, the positive relationship between the degree of interpolated learning and the forgetting of the original task, found in previous studies, may have arisen simply because the higher degree of interpolated learning induced greater retrieval practice on the interpolated task. If this line of reasoning is right, this positive relationship should disappear if the amount of retrieval practice is the same across the different acquisition degrees, or if there is no retrieval practice on the interpolated task at all.

This study reports two free-recall experiments in which the influence of the degree of original and interpolated learning on the amount of retroactive interference is investigated again. These experiments were designed to meet the criteria emphasized above. First, the recall of the original task is free of floor effects, and, second, the amount of retrieval practice on the interpolated task either is the same across acquisition degrees or is eliminated altogether, thus separating the possible effect of the degree of learning from the possible effect of retrieval practice. Different study times were used for producing different degrees of learning (Ratcliff, Clark, \& Shiffrin, 1990; Slamecka \& McElree, 1983; Wickelgren, 1977). Unlike the anticipation procedure, this procedure makes repeated retrieval of the learning material unnecessary.

\section{EXPERIMENT 1}

\section{Method}

\section{Subjects \\ Two hundred and twenty-five psychology students at the University of Regensburg participated in the experiment.}

\footnotetext{
Material

The material consisted of 125 items. One hundred of these items were different conceptually categorizable pairs (e.g., translated into English: apple-pear, cello-trombone, etc.). The remaining 25 items were "singles"- that is, items that are not categorizable in an obvious manner, either mutually or with regard to the pairs. The pairs and the singles were largely taken from the categories of Battig and Montague (1969), although some categories not found in Battig and Montague were also included in the lists (Scheithe \& Bäuml, 1995). Strong exemplars were
}

chosen from each category. The two items in each pair were therefore highly likely to be associated with each other. The 125 items were randomly assigned to five lists, with the restriction that in every list there had to be 10 pairs and 5 singles. Thus, every list consisted of 25 items. The same material was used for all three experimental conditions.

\section{Design}

The experiment included three experimental conditions: the high-high, the low-high, and the high-low condition. In all three conditions, one learning list and zero to four interpolated lists were presented to five different groups of subjects, with 15 subjects per group. In the highhigh condition, all items from all lists were presented at an exposure rate of $5 \mathrm{sec}$ per item. In the low-high condition, the items of the first list were presented for $2 \mathrm{sec}$ per item, and the items from the interpolated lists were presented for $5 \mathrm{sec}$. This condition addresses the issue of whether the degree of original learning affects the amount of retroactive interference. In the high-low condition, the items on the interpolated lists were presented for $2 \mathrm{sec}$ per item, and the items on the first list were presented for $5 \mathrm{sec}$. This condition addresses the issue of whether the degree of interpolated learning affects the amount of retroactive interference.

\section{Procedure}

At the beginning of each experimental session, the items within each list were mixed randomly, with the restriction that the pairs were presented with no intervening items. The items were presented individually on a computer screen. After the presentation of each complete list, the subjects were given $90 \mathrm{sec}$ to write down the items from this list. After this recall period and a break of $60 \mathrm{sec}$, the session continued with the next list. After all of the lists were presented, a final free-recall test was given in which the subjects attempted to recall the items from all of the previous lists in any order. The subjects were given a minimum of $5 \mathrm{~min}$ for this final written recall, with additional time allowed. This procedure is similar to the one used by Tulving and Psotka (1971), Riefer and Batchelder (1988), and Bäuml (1991).

\section{Results}

\section{High-High Condition Versus Low-High Condition}

Learning. The manipulation of study time on the original list had an effect on the degree to which it was learned. In the high-high condition, recall performance without interpolation was 19.5 items; in the low-high condition, it was 15.9 items. This difference was reliable $\left[F(1,28)=13.6, M S_{\mathrm{e}}=6.7, p=.001\right]$, indicating that degree of original learning was successfully manipulated. Because of the identical study times for all four interpolated lists of the two experimental conditions, one would expect to find the same degree of learning for these lists. ${ }^{2}$ An analysis of variance (ANOVA) for the data of the five-list groups of the two experimental conditions confirmed this expectation: There was no main effect of condition $[F(1,28)<1]$, no main effect of list $\left[F(3,84)=1.6, M S_{\mathrm{e}}=3.6, p=.20\right]$, and no interaction between the two factors $[F(3,84)<1]$. Mean performance on these interpolated lists was 19.1 items.

Forgetting. For all five interpolation levels of the highhigh condition and the low-high condition, the final recall of the original list was analyzed. Figure 1A shows the data. Inspection of the graph suggests that both study time on the original list and interpolation influenced recall performance, demonstrating the presence of different acquisition degrees and of forgetting. More important, the two forgetting functions look fairly parallel, which indicates that the difference in recall performance caused 

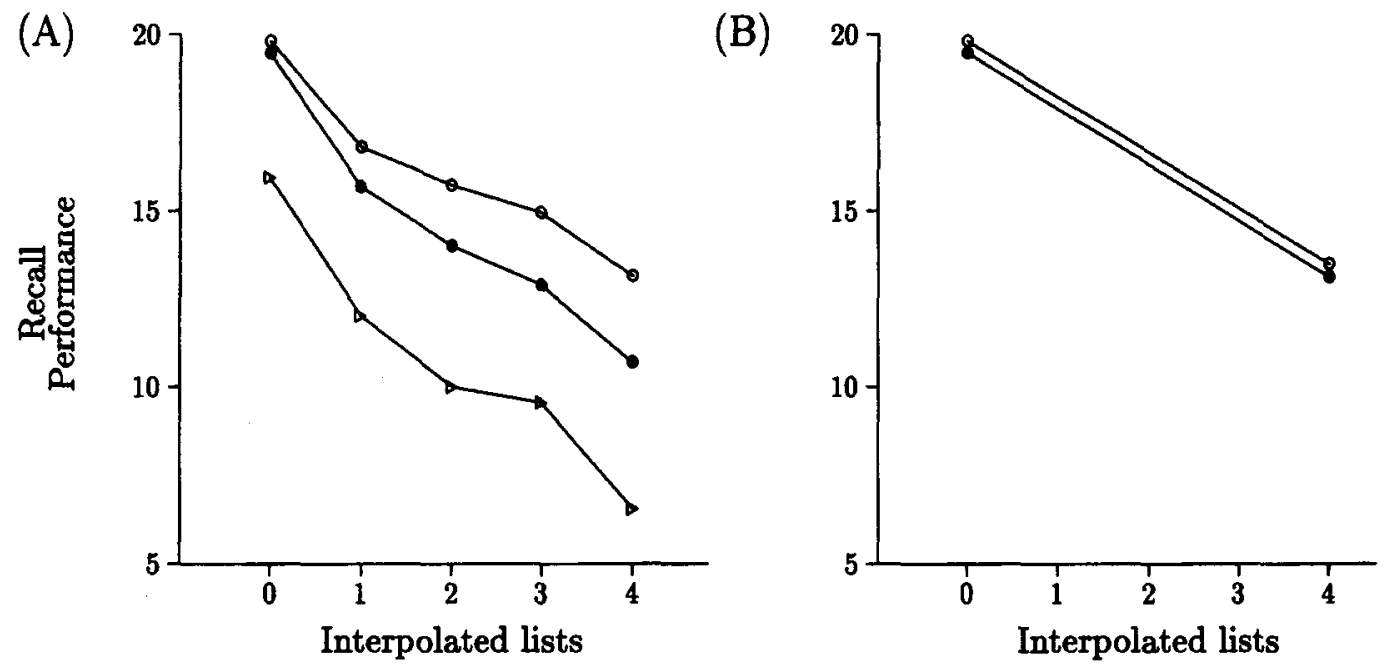

Figure 1. Free recall of the original list as a function of the number of interpolated lists and the degree of original and interpolated learning in Experiment 1 (A) and Experiment 2 (B). $\bullet=$ high-high condition (high degree of both original and interpolated learning). $O=$ high-low condition (high degree of original and low degree of interpolated learning). $\triangleright=$ low-high condition (low degree of original and high degree of interpolated learning).

by different study times on the original list remained about the same across interpolation levels. An ANOVA confirmed this visual impression: While there were highly significant main effects of study time $[F(1,140)=38.2$, $\left.M S_{\mathrm{e}}=13.5, p<.001\right]$ and interpolation level $[F(4,140)=$ $\left.25.1, M S_{\mathrm{e}}=13.5, p<.001\right]$, the value of the critical interaction was found to be far from significant $[F(4,140)<1]$.

\section{High-High Condition Versus High-Low Condition}

Learning. Because of the identical study times on the original list of the two experimental conditions, one would expect to find the same degree of learning for these lists. In fact, recall performance without interpolation did not differ reliably $[F(1,28)<1]$, with a recall of $19.5 \mathrm{i}^{+} \mathrm{ems}$ in the high-high condition and 19.8 items in the highlow condition. The manipulation of study time on the interpolated lists of the two experimental conditions had an effect on the degree to which they were learned. The data of the five-list groups of the two experimental conditions showed a mean performance of 19.4 items for the interpolated lists in the high-high condition and 15.4 items for those in the high-low condition. An ANOVA for these data revealed the expected main effect of study time $[F(1,28)=$ $\left.11.1, M S_{\mathrm{e}}=41.8, p=.002\right]$, but no main effect of list $\left[F(3,84)=1.1, M S_{\mathrm{e}}=4.5, p=.36\right]$ and no interaction between the two factors $[F(3,84)<1]$. Thus, degree of interpolated learning was successfully manipulated.

Forgetting. Figure 1A shows the final recall of the original list in the high-high condition and the high-low condition for all five interpolation levels. Inspection of the graph suggests two things. First, interpolation had an effect on recall in both conditions, indicating the presence of forgetting. Second, on the basis of a roughly equal performance when there was no interpolation, performance in the high-low condition was less impaired by interpolation than was performance in the high-high condition. The difference in recall across the two conditions was 1.2 items after one interpolated list, increasing up to 2.5 items after four interpolated lists. An ANOVA confirmed that, with interpolation, both the study time of the interpolated lists $\left[F(1,112)=7.7, M S_{\mathrm{e}}=13.3\right.$, $p=.006]$ and the interpolation level $[F(3,112)=7.5$, $\left.M S_{\mathrm{e}}=13.3, p<.001\right]$ reliably influenced recall performance. The small tendency for a larger difference in case of a higher interpolation level did not reach significance $[F(3,112)<1] .^{3}$

\section{Discussion}

The degree of original and interpolated learning was successfully manipulated in this experiment and retroactive interference was present. Also, recall of the original task was reasonably free of floor effects in all three experimental conditions, thus meeting the third basic precondition to examine the role of original and interpolated learning on retroactive interference.

Upon comparison, the forgetting functions of the high-high condition and the low-high condition were discovered to be parallel. In fact, the difference in recall performance (caused by the degree of original learning without interpolated material) was maintained when interpolation was introduced. The amount of forgetting, therefore, did not depend on the degree of original learning. This result generalizes Slamecka and McElree's (1983) findings for normal forgetting to retroactive interference. It also confirms the view proposed above that the results from earlier studies, in which dependencies between retroactive interference and the degree of original learning had been found, probably were due to floor effects.

A comparison of the forgetting functions of the high-high condition and the high-low condition revealed that the functions are different across conditions. In fact, recall performance after a low degree of interpolated learning was significantly higher than was recall performance after a high degree of interpolated learning. The amount of forgetting, therefore, did depend on the degree of interpolated learning. This pattern of results parallels those from all previous studies, which also found a positive relationship between the degree of interpolated learning and the amount of forgetting of the original task. Nevertheless, as is argued in the next paragraph, it might still be premature to conclude that it was the degree of interpolated learning per se that caused this positive relationship. 
To corroborate that an increasing study time really improved performance on the interpolated lists, the subjects had to do a recall test after studying each interpolated list. These recall tests, however, also engaged the subjects in retrieval practice on those lists. It is therefore crucial for this experiment, and the conclusions that are drawn from its results, that the amount of retrieval practice was the same across the different degrees of interpolated learning.

This supposition might be wrong. In fact, the subjects recalled more items in the high-high condition than in the high-low condition during these interpolated tests. Thus, the subjects in the high-high condition may have had more occasions to suppress first-list items than did the subjects in the high-low condition (Anderson et al., 1994; Anderson \& Spellman, 1995). Moreover, the final recall test required the subjects to remember all items from all study lists in any order. Since the subjects in the high-high condition remembered more items from the interpolated lists than did the subjects in the high-low condition, they also recalled more items from these lists on this final recall test. As a result, there may have been more output interference for first-list items in the high-high condition than in the high-low condition (Smith, 1971). Each of these two factors may have contributed to the lower recall in the highhigh condition. Thus, not only differences in the degree of interpolated learning but also differences in the amount of retrieval practice and of output interference could have caused the different amounts of forgetting. Experiment 2 was conducted to examine these objections.

\section{EXPERIMENT 2}

\section{Method}

\section{Subjects}

Eighty psychology students at the University of Regensburg participated in the experiment.

\section{Material \\ The material was identical to that used in Experiment 1.}

\section{Design}

The experiment included two experimental conditions: the high-high condition and the high-low condition. In both conditions, one learning list and four interpolated lists were presented to 40 subjects. As in Experiment 1 , in the high-high condition, all items from all lists were presented at an exposure rate of $5 \mathrm{sec}$ per item. In the high-low condition, the items on the interpolated lists were presented for $2 \mathrm{sec}$ per item, and the items on the first list were presented for $5 \mathrm{sec}$ each.

\section{Procedure}

The procedure was identical to the one used in Experiment 1, with two exceptions. First, the recall tests after each interpolated list were eliminated and, instead, the subjects were engaged in a $90-\mathrm{sec}$ distractor task. Second, in the final recall test, the subjects were directed to recall the items from the first list first. The subjects were given a minimum of $3 \mathrm{~min}$ for this first-list recall, with additional time allowed. It was stressed that only items from the first list should be recalled. This recall was followed immediately by the recall of the second list, the recall of the third list, and so on. The same procedure was used for the recall of these four interpolated lists as for the recall of the first list. Again, for each single list, it was stressed that only items from this list should be recalled.

\section{Results}

\section{Learning}

As opposed to the procedure in Experiment 1, the procedure in Experiment 2 no longer allowed the direct examination of the degree of interpolated learning across the two experimental conditions. However, since the learning method was similar to the one used in Experiment 1, it was assumed that the manipulation of the degree of interpolated learning would be comparable to that in Experiment 1 (see Discussion below).

\section{Forgetting}

For each of the two experimental conditions, the original list items that were recalled during the first-list final recall were analyzed. Figure 1B shows the recall performance in the high-high condition and the high-low condition after the four interpolated lists, together with the two conditions' no-interpolation data from Experiment 1. Inspection of the graph suggests that the different study times for the interpolated lists did not have an effect on recall performance. In fact, recall performance was 13.1 items in the high-high condition and 13.5 items in the high-low condition. An ANOVA confirmed that this difference is far from being reliable $[F(1,78)<1]{ }^{4}$

\section{Discussion}

Experiment 2 was designed to completely separate the effect of interpolated learning from the possible effect of retrieval factors. Whereas Experiment 1 may still have confounded the degree of interpolated learning and the amount of retrieval practice and output interference to some extent, this source of bias was eliminated in Experiment 2 by excluding the recall tests after each interpolated list and directing the subjects in the final recall tests to recall the original list first.

As opposed to the results of Experiment 1, a comparison of the forgetting functions between the high-high condition and the high-low condition in Experiment 2 revealed no difference across conditions. Indeed, recall performance after a high degree of interpolated learning was practically identical to recall performance after a low degree, indicating that the amount of retroactive interference did not depend on the degree of interpolated learning.

Although the manipulation of the degree of interpolated learning in Experiment 2 could not be examined directly, a reasonable estimate of this manipulation can be provided by comparing the final recall performance for the second list in the high-high condition with that in the high-low condition. For this second list, the two conditions match both with respect to a possible proactive interference that might result from the prior learning of the original list and, on the basis of the results of Experiment 2 , with respect to a possible output interference that might result from the prior recall of the original list. Now, if the amount of retroactive interference does not depend on the degree of original learning (see Experiment 1) and does also not depend on the degree of interpolated learning (see Experiment 2), then the difference that is found in the final recall of the second list reflects the manipulation of degree of interpolated learning for this list. Final recall performance for the second list was 12.1 items in the high-high condition and 5.4 items in the high-low condition $\left[F(1,78)=38.1, M S_{\mathrm{e}}=23.0, p<.001\right]$, indicating a considerable manipulation of the degree of interpolated learning in this experiment. ${ }^{5}$

The result that degree of interpolated learning did not influence the amount of retroactive interference in Experiment 2 suggests two things. First, it was not the degree of interpolated learning per se that caused the larger amount of interference for the condition with the higher degree of interpolated learning in Experiment 1. Rather, it was probably the different amount of retrieval practice and of output interference across the two acquisition conditions that mediated the effect. Second, since all previous studies used the anticipation procedure during acquisition of the interpolation task, thus introducing a different amount of retrieval practice for the different acquisition degrees, the consistent finding of a positive relationship between the degree of interpolated learning and the ability to recall the original list items may reflect the effect of different amounts of retrieval practice. If true, it was not difference in the degree of interpolated learning that caused the positive relationship in previous studies.

\section{CONCLUSIONS}

First, the present results indicate that the degree of original learning has no substantial influence on the amount of retroactive interference exerted by later lists, which is consistent with other work on normal forgetting. More surprising, they even suggest that the degree of interpo- 
lated learning also has no influence on retroactive interference. These invariances establish important empirical restrictions for models of forgetting. The finding that greater interpolated learning does not increase the amount of retroactive interference challenges forgetting models that incorporate strength-dependent competition mechanisms (Mensink \& Raaijmakers, 1988) and seems inconsistent with similar accounts of other empirical phenomena, such as part-list cuing impairment (Rundus, 1973).

Second, the different pattern of results in Experiments 1 and 2 reveals nicely how retrieving some material may substantially inhibit the recall of (previously learned) related material. By using quite a different experimental paradigm than has been used in prior research (Anderson et al., 1994; Roediger, 1974, 1978; Smith, 1971), this finding provides another striking demonstration of the important role of retrieval-induced forgetting in recall from long-term memory.

Finally, in the present study, lists of categorizable item pairs and single items were presented to subjects. Many of the previous studies, however, used paired-associate study lists, in which cue-target associations are presented to subjects, and each original target item has a very specific interpolated item with which it competes. A priori, it is possible that, even though there is no evidence for a relationship between the degree of interpolated learning and the amount of retroactive interference in this study, a positive relationship may be found using the pairedassociate method. Future studies must clarify whether the present pattern of results generalizes to this method. ${ }^{6}$

\section{REFERENCES}

Anderson, M. C., Bjork, R. A., \& BJork, E. L. (1994). Remembering can cause forgetting: Retrieval dynamics in long-term memory. Journal of Experimental Psychology: Learning, Memory, \& Cognition, 20, 1063-1087.

Anderson, M. C., \& Spellman, B. A. (1995). On the status of inhibitory mechanisms in cognition: Memory retrieval as a model case. Psychological Review, 102, 68-100.

BARNES, J. M., \& UNDERWOOD, B. J. (1959). "Fate" of first-list associations in transfer theory. Journal of Experimental Psychology, 58, 95-105.

BATCHELDER, W. H., \& RIEFER, D. M. (1986). The statistical analysis of a model for storage and retrieval processes in human memory. British Journal of Mathematical \& Statistical Psychology, 39, 129-149.

Battig, W. F., \& Montague, W. E. (1969). Category norms for verbal items in 56 categories: A replication and extension of the Connecticut category norms. Journal of Experimental Psychology, 80, 1-46.

BÄUML, K.-H. (1991). Experimental analysis of storage and retrieval processes involved in retroactive inhibition: The effect of presentation mode. Acta Psychologica, 77, 103-119.

BÄUML, K.-H. (in press). A Markov model for measuring storage loss and retrieval failure in retroactive inhibition. Acta Psychologica.

BRIGGS, G. E. (1957). Retroactive inhibition as a function of the degree of original and interpolated learning. Journal of Experimental Psychology, 53, 60-67.

DaPolito, F. J. (1966). Proactive effects with independent retrieval of competing responses. Unpublished doctoral dissertation, Indiana University.

EsTes, W, K. (1988). Human learning and memory. In R. C. Atkinson, R. J. Hermstein, G. Lindzey, \& R. D. Luce (Eds.), Stevens' Handbook of experimental psychology (2nd ed., pp. 351-415). New York: Wiley.

LoFTUS, G. R. (1985). Evaluating forgetting curves. Journal of Experimental Psychology: Learning, Memory, \& Cognition, 11, 397-406.

MENSINK, J.-G., \& RaAimakers, J. G. W. (1988). A model of interference and forgetting. Psychological Review, 95, 434-455.

Postman, L., \& Riley, D. A. (1959). Degree of learning and interserial interference in retention: A review of the literature and an experimental analysis. University of California Publications in Psychology, 8 271-396.

RatClifF, R., Clark, S. E., \& ShIFFrin, R. M. (1990). The list-strength effect: 1. Data and discussion. Journal of Experimental Psychology. Learning, Memory, \& Cognition, 16, 163-178.

RIEFER, D. M., \& BATCHELDER, W. H. (1988). Multinomial modeling and the measurement of cognitive processes. Psychological Review, 95, 318-339.

ROEDIGER, H. L., III (1974). Inhibiting effects of recall. Memory \& Cognition, 2, 261-269.
Roediger, H. L., III (1978). Recall as a self-limiting process. Memory \& Cognition, 6, 54-63.

Rundus, D. (1973). Negative effects of using list items as recall cues Journal of Verbal Learning \& Verbal Behavior, 12, 43-50.

SCHEITHE, K., \& BÄUML, K.-H. (1995). Deutschsprachige Normen für Vertreter von 48 Kategorien [German normative data for representatives of 48 categories]. Sprache \& Kognition, 14, 39-43.

SLAMECKA, N. J. (1960). Retroactive inhibition of connected discourse as a function of practice level. Journal of Experimental Psychology, 59, 104-108.

SLAMECKA, N. J. (1985). On comparing rates of forgetting. Journal of Experimental Psychology: Learning, Memory, \& Cognition, 11, 812-816.

SLAMECKA, N. J., \& MCELREE, B. (1983). Normal forgetting of verbal lists as a function of their degree of learning. Journal of Experimental Psychology: Learning, Memory, \& Cognition, 9, 384-397.

SMITH, A. D. (1971). Output interference and organized recall from long-term memory. Journal of Verbal Learning \& Verbal Behavior, $10,400-408$.

Tulving, E., \& PsotKa, J. (1971). Retroactive inhibition in free recall: Inaccessibility of information available in the memory store. Journal of Experimental Psychology, 87, 1-8.

WiCKELGREN, W. A. (1977). Learning and memory. Englewood Cliffs, NJ: Prentice-Hall.

\section{NOTES}

1. To come to the conclusion that the degree of learning does not influence the amount of forgetting, Slamecka and McElree (1983) assumed the rates of forgetting to be equal for two acquisition degrees, if the forgetting functions of the two conditions were (vertically) parallel. Although this definition of equal forgetting is, to a certain degree, arbitrary (Bäuml, in press; Loftus, 1985), it is often regarded to be the most natural one (Estes, 1988; Slamecka, 1985) and thus has also been adopted in this study

2. This expectation is also based on Tulving and Psotka's (1971) finding that there is no proactive interference in this situation.

3. The data of Experiments 1 and 2 were also analyzed by means of Batchelder and Riefer's (1986) multinomial storage-retrieval model. On the basis of certain assumptions, this model enables a separate analysis of storage and retrieval processes. The analysis showed that the degree of original learning affected both storage and retrieval capacities, whereas interpolation-high or low-influenced only retrieval but not storage capacities, which is consistent with prior research on the source of retroactive interference (Bäuml, 1991; Riefer \& Batchelder, 1988). The influence of the degree of interpolated learning found in Experiment 1 was thus due to different impairments in retrieval capacities for the two acquisition degrees: A high degree of interpolated learning impaired retrieval of the original list to a greater extent than did a low degree of interpolated learning.

4. Some subjects also recalled original list items during the final recall of the interpolated lists. On average, 0.4 items from the original list were recalled during this postinitial period in the high-high condition and 0.3 items in the high-low condition. Thus, the recall of original list items after the initial recall period was very rare and was not substantially different between the two conditions. If these items had been included in the analysis, the pattern of outcomes, therefore, would not have changed.

5. A similar pattern of results was found for the final recall of the third list (12.3 items in the high-high condition vs. 5.9 items in the highlow condition), the fourth list (13.0 items vs. 7.8 items), and the fifth list (10.1 items vs. 7.3 items). However, note that-as opposed to the final recall of the second list--for the final recall of these last three lists, the two conditions no longer match with respect to a possible proactive interference and a possible output interference that may result from the prior learning and prior recall of other lists. Indeed, the data are probably contaminated by these two sources of interference.

6. DaPolito (1966) investigated the role of the degree of prior learning on the amount of pmactive interference using a paired-associate task. He found that the degree of prior learning did not affect the recall of the target items (see Riefer \& Batchelder, 1988, for a multinomial analysis of this data set).

(Manuscript received January 20, 1995; revision accepted for publication April 3, 1996.) 